\title{
New measurements and quantitative analysis of electron backscattering in the energy range of neutron $\beta$-decay
}

\author{
J. W. Martin, ${ }^{1,4}$ J. Yuan, ${ }^{1}$ M. J. Betancourt, ${ }^{1}$ B. W. Filippone, ${ }^{1}$ S. A. Hoedl, ${ }^{3}$ T. M. Ito, ${ }^{1, *}$ B. Plaster, ${ }^{1}$ and A. R. Young ${ }^{2}$ \\ ${ }^{1}$ W. K. Kellogg Radiation Laboratory, California Institute of Technology, Pasadena, California 91125, USA \\ ${ }^{2}$ North Carolina State University, Raleigh, North Carolina 27695, USA \\ ${ }^{3}$ CENPA, University of Washington, Seattle, Washington 98195, USA \\ ${ }^{4}$ Physics Department, University of Winnipeg, Winnipeg, Manitoba, Canada R3B 2E9
}

(Received 5 August 2005; published 25 January 2006)

\begin{abstract}
We report on the first detailed measurements of electron backscattering from plastic scintillator targets, extending our previous work on beryllium and silicon targets. The scintillator experiment posed several additional experimental challenges associated with charging of the scintillator target, and those challenges are addressed in detail. In addition, we quantitatively compare the energy and angular distributions of this data, and our previous data, with electron transport simulations based on the GEANT4 and PENELOPE Monte Carlo simulation codes. The PENELOPE simulation is found globally to give a superior description of the data. Such information is crucial for a broad array of weak-interaction physics experiments, where electron backscattering can give rise to the dominant detector-related systematic uncertainty.
\end{abstract}

DOI: 10.1103/PhysRevC.73.015501

PACS number(s): 23.40.-s, 29.30.Dn, 34.80.Bm

\section{INTRODUCTION}

Our previous work on backscattering [1] from beryllium and silicon extended the work of others [2-4] to energies relevant for nuclear physics applications. We further extend this work to a more relevant target, organic scintillator. The main experimental challenge arose from the fact that the target material is not conducting, necessitating mitigation of effects due to charging in order to accurately sense currents.

\section{EXPERIMENT OVERVIEW}

As for our previous measurements, the experiment consisted of an electron gun and a scattering chamber containing a movable target [1]. Two modes of acquiring backscattering data were used. In one mode a silicon detector was used to detect the energy and angle of backscattered electrons. In a second higher-current mode, the electrical current due to the backscattered electrons incident on the chamber walls was measured. These two modes were referred to as silicon detector mode and current integration mode, respectively.

The plastic scintillator targets used for the experiment were obtained from Eljen Technologies [5]. The type of plastic scintillator used was EJ-204. The density of EJ-204 is $1.032 \mathrm{~g} / \mathrm{cm}^{3}$, with the dominant elemental composition being $5.21 \times 10^{22} \mathrm{H}$ atoms $/ \mathrm{cm}^{3}$, and $4.74 \times 10^{22} \mathrm{C}$ atoms $/ \mathrm{cm}^{3}$. A sample of plastic scintillator was coated with a $500 \AA$ thick layer of aluminum via evaporation. Targets of a suitable size and shape were then cut from this sample, with care taken to preserve the aluminized front face. The resulting targets were 1 in. $\times 1$ in. square, $3 \mathrm{~mm}$ thick, with a thinner tab of aluminized plastic projecting from the top for suspension from a central rotatable feedthrough.

\footnotetext{
*Present address: Los Alamos National Laboratory, Los Alamos, NM 87545.
}

As before, the energy and angular distribution of the backscattered electrons was measured using a $25 \mathrm{~mm}^{2}$ active area silicon detector. The energy resolution of the silicon detector system was typically $\sigma=4.3 \mathrm{keV}$, and was independent of energy.

\section{BACKSCATTERING MEASUREMENTS}

Backscattering measurements were performed for normal incidence upon the target. Measurements were performed for incident electron energies of 43.5, 63.9, 83.8, 104, and $124 \mathrm{keV}$. For each energy, both silicon detector mode and current integration mode measurements were taken.

\section{A. Silicon detector mode}

The quantities which varied for the silicon detector measurements conducted at a particular electron beam energy $E_{\text {beam }}$ were the detection angle $\theta$, and the energy $E$ the backscattered electron deposited in the silicon detector. The angle $\theta$ was defined relative to the surface normal vector of the material, so that $\theta=0$ degrees corresponds to a backscattering event where the electron went directly back along the incident beam direction. The dimensionless energy $q=$ $E / E_{\text {beam }}$ will also be used.

For silicon detector mode, the systematic uncertainty is unchanged from our previous work, resulting in an average $12 \%$ normalization systematic for each angular setting of the detector. However, we specifically addressed current detection and charging of the target, as will now be discussed.

To ensure the reliability of measurements of current, we compared the measurements of two different, carefully calibrated current integrators against one another for identical experimental conditions, and in turn cross-compared those measurements against two calibrated picoammeters. The measurements were consistent at the $3 \%$ level.

The possible effects of charging and incomplete current detection were monitored by observing scintillation light from 
the electron beam as it struck the scintillator target, on a camera mounted behind the target outside a viewport in the chamber (at $\left.\theta=180^{\circ}\right)$. Over the course of taking a complete angular range of data for a particular incident beam energy, the brightness of the scintillation light did not visibly change with time. Previous tests with uncoated scintillator, or scintillator coated poorly with graphite, had shown that the emanation of scintillation light from the target would eventually cease, indicating that the electron beam had been diverted away from the central spot on the target. Also discharges would be seen due to arcing from the face of the scintillator to the conducting target rod. No such effects could be seen with the Al-coated scintillator target. On leaving the beam on the target for long periods of time, no change in the sensed current was seen at the $1 \%$ level. To also search for charging, the electron beam could be switched off and on rapidly by inserting a Faraday cup upstream of the chamber. Upon restoration of the beam, the current sensed by the target was found to agree with the value before intercepting the beam with the Faraday cup to the $1 \%$ level.

The effect of the aluminum layer can be estimated by comparing the thickness of the Al layer to the mean range of the electrons. The mean range for $43.5 \mathrm{keV}$ electrons in plastic scintillator is $30 \mu \mathrm{m}$ [6], which is three orders of magnitude larger than the thickness of the Al layer. This implies that scattering from the $\mathrm{Al}$ is suppressed below the $1 \%$ level, even for the lowest energy incident electrons reported in this work. Both estimates were confirmed in Monte Carlo studies of scattering from thin layers [7]. For the silicon detector data, the effects of the Al layer were therefore neglected.

As a cross check of the reproducibility, dead-time, and current detection uncertainties due to charging, runs were taken for various beam currents. The normalized yield for these runs was found not to vary outside the previously quoted $7 \%$ reproducibility uncertainty.

For completeness, a catalog of the dominant systematic uncertainties considered for silicon detector mode is displayed in Table I.

Aside from target deterioration, which will be discussed in the next section, these systematic uncertainties were described in detail in our previous work [1]. The effects listed in the upper portion of Table I refer to results for the observable $\frac{1}{N_{e}} \frac{d N}{d \Omega d q}$, and average $12 \%$. The effects in the lower portion of

TABLE I. Dominant systematic uncertainties for silicon detector mode.

\begin{tabular}{lc}
\hline \hline Effect & Uncertainty \\
\hline Reproducibility & $7 \%$ \\
Active area & $4 \%$ \\
Finite beam spot & $5 \% \times \sin \theta$ \\
Dead time & $3 \%$ \\
Alignment & $2 \%$ \\
Current detection & $3 \%$ \\
Target deterioration & $1 \%$ \\
Extrapolation over $q$ & $6-20 \%$ \\
Extrapolation over $\theta$ & $4 \%$ \\
Total & $12-23 \%$ \\
\hline \hline
\end{tabular}

Table I refer to extrapolation uncertainties encountered when integrating these data over $q$ and/or $\theta$, and these must be added in quadrature to those above when considering our results for $d \eta / d \Omega$ and $\eta$ from silicon detector mode. For extrapolation over $q$, the uncertainty is larger for lower beam energy, as the finite detection threshold becomes more important; hence the $20 \%$ value refers to beryllium at $43.5 \mathrm{keV}$ incident energy, since the beryllium data are more peaked at lower $q$, while the $6 \%$ value refers to data taken with $124 \mathrm{keV}$ incident energy. Considering the range given for the total systematic uncertainty, the lower bound (12\%) refers to all observations of $\frac{1}{N_{e}} \frac{d N}{d \Omega d q}$, while the upper bound (23\%) refers specifically to the extraction of $\eta$ from beryllium at $43.5 \mathrm{keV}$, where extrapolation uncertainties dominate.

For these experiments, the silicon detector threshold varied from 10 to $18 \mathrm{keV}$ depending on noise conditions at the time of any given data-taking run.

Effects due to backgrounds from $\mathrm{X}$ rays, and multiply backscattered electrons were also discussed in detail in our previous work. Multiply backscattered electrons resulted in systematic uncertainties at the 3\%-5\% level at $q=0.2$ for the highest beam energy considered. But this contribution to the systematic uncertainty is below $1 \%$ above $q=0.3$, and is negligible for lower beam energies.

\section{B. Current integration mode}

For each beam energy setting, current integration mode measurements were also performed.

The largest potential systematic effect, which was previously uncontrolled, arose from deterioration of the scintillator target by the electron beam. Over the course of an hour, running at beam currents of tens of $\mathrm{nA}, \eta$ was observed to steadily increase with time, plateauing at a value typically $15 \%$ larger than its initial value. The transition to larger $\eta$ was found to occur more rapidly with higher beam currents and higher beam energies. The glow of the beam spot on the target was also monitored on the video camera and found to decrease in brightness in a correlated way. The brightness was found not to recover after leaving the scintillator in vacuum over several days, ruling out charging/discharging of the bulk scintillator. Upon removal of an affected target from the vacuum chamber, a small brownish spot within the scintillator could be observed, with no obvious deterioration of the mirror-like aluminized front face. We believe the discoloration, reduction in scintillation light, and increase in $\eta$ are symptoms of a chemical change in the scintillator, possibly resulting in the liberation of hydrogen. This would increase the relative carbon content and hence $\eta$.

To control this potential systematic, new scintillator targets were used and were exposed to electron beams with currents less than $1 \mathrm{nA}$. The targets were exposed to beam for the minimum time possible for currents to be sensed accurately using picoammeters. This limited the contribution to the systematic uncertainty to less than $1 \%$, based on the slope measured during the prior hour-long measurements.

A dependence on beam current at the $3 \%$ level had been seen for our previous current integration mode data on $\mathrm{Be}$ and $\mathrm{Si}$ 
target [1]. For those data, the current dependence was attributed to charging of various components in the chamber. As our new studies were done generally at lower beam currents, we expect this contribution to be smaller, but retain the pessimistic upper limit of $3 \%$.

In order to characterize and control the effects of low-energy secondary electrons (for our work, defined to be below $50 \mathrm{eV}$ in energy), a wire mesh cage was inserted into the chamber and held at $-55 \mathrm{~V}$. From an electrostatic model of the electric potential in the chamber, this resulted in a minimum potential wall of $-50 \mathrm{~V}$ between the target and the chamber. For the purposes of modelling these measurements, the integrated backscatter current was therefore taken to be due to all electrons emanating from the target with greater than $50 \mathrm{eV}$.

The wire mesh cage was referred to as "the grid" consistent with terminology used in previous backscattering literature. The grid was constructed from a thin copper rod bent into a cylindrical shape. Steel wire was wound on the resultant frame in an end-over-end pattern resulting in vertical wires equally spaced running down the sides of the cylindrical shape. The resultant cylindrical wire cage resembled a bird cage which enclosed the target. In this way, biasing the grid at negative potential prevented secondaries from traveling from the chamber walls to the target and vice versa.

However, as with our previous work, the insertion of the grid resulted in systematic effects due to incomplete compensation for secondaries and due to effects of backscattered electrons striking the grid itself.

A residual correction due to a piece of conducting target rod penetrating the top of the grid must be applied. The rod subtended a small but finite solid angle viewing the beam spot on the target. High-energy backscattered electrons could strike that portion of the rod, resulting in secondary electron production. Secondary electrons created on that portion of the rod would not be suppressed by the grid and would be collected on the target. This resulted in a residual dependence of the apparent $\eta$ on target voltage, which could be corrected. The correction gave rise to a $7 \%$ contribution to the systematic uncertainty in the previous work, and was the dominant uncertainty. For this work, the contribution was reduced to $3 \%$ by reducing the solid angle subtended by the relevant portion of the target rod. This reduced the size of the correction, and hence the systematic uncertainty. Detailed analytical calculations of the effect of the solid angle and its variation with, e.g., distance of the beam spot from the top of the target close to the target rod gave additional confidence in this uncertainty. As before, secondaries created on the grid were characterized by monitoring the grid current, and contributed at the $1 \%$ level.

A potential systematic effect arises due to differences in backscatter yields above $50 \mathrm{eV}$ because of the presence of the $\mathrm{Al}$ coating on the target. Electrons with energies above $100 \mathrm{eV}$ could not be adequately assessed via altering the voltage on the grid, while from the electron transport arguments presented earlier, only for energies above about $10 \mathrm{keV}$ can the effects of the Al layer be argued to be negligible. From similar considerations to the extrapolation uncertainty for silicon detector mode, we limit possible effects of the Al coating in this energy range to the $6 \%$ level.
TABLE II. Dominant systematic uncertainties for current integration mode for scintillator target data.

\begin{tabular}{lc}
\hline \hline Effect & Uncertainty \\
\hline Target rod correction & $3 \%$ \\
Grid secondaries & $1 \%$ \\
Reproducibility & $5 \%$ \\
Current dependence & $3 \%$ \\
Target deterioration & $1 \%$ \\
Al coating effects & $6 \%$ \\
Total & $9 \%$ \\
\hline \hline
\end{tabular}

The dominant systematic uncertainties for currentintegration mode results for our new scintillator target data are listed in Table II.

\section{RESULTS}

\section{A. Silicon detector mode}

The normalized, background-subtracted spectra accumulated for various detector angles for $124 \mathrm{keV}$ electrons normally incident on a scintillator target is shown in Fig. 1.

The data are plotted as a function of the dimensionless energy $q$. On the vertical axis, $\frac{1}{N_{e}} \frac{d N}{d q d \Omega}$, the number of counts per incident electron, per unit $q$, per unit solid angle is plotted. In the absence of the effects of detector response (resolution

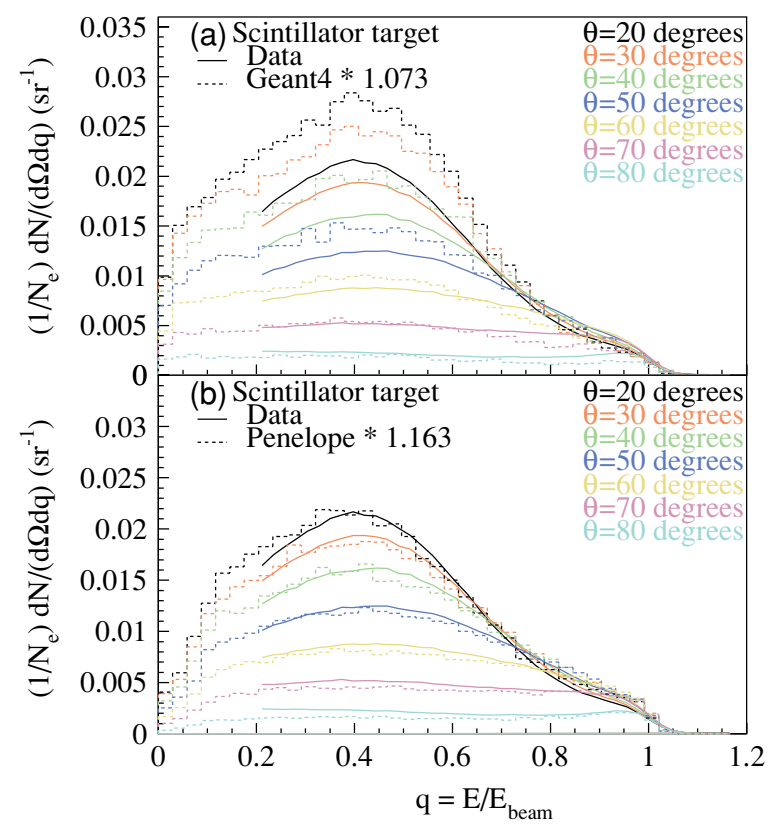

FIG. 1. (Color online) Normal incidence backscattering from scintillator target at $E_{\text {beam }}=124 \mathrm{keV}$. Curves represent data taken with silicon detector. Histogram is Monte Carlo simulation based on (a) GEANT4, and (b) PENELOPE. Systematic uncertainty in the normalization of the data is estimated to be $12 \%$ on average, ranging from $11 \%$ at small angles to $15 \%$ at large angles. A scale factor of 1.073 is applied to the (a) GEANT4 simulation. In (b), the factor is 1.163 for PENELOPE. 
and backscattering), this would be the normal-incidence backscattered fraction per unit $q$, per unit solid angle.

Monte Carlo simulations of backscattering were carried out with custom codes based on the GEANT4 [8] and PENELOPE [9] toolkits. The custom aspects of the simulation codes were described in Ref. [1], for example, their handling of backscattering from the silicon detector itself and the detector response function (relating to this particular observable). Figure 1 compares the data with the results of these two codes. In each case, a scale factor is applied to the Monte Carlo. The scale factor was determined from a fit to the data, which is described in Sec. V. As for our previous work, GEANT4 systematically underestimates the peak in the data near $q=0.95$. However the positions in $q$ of the low-energy and elastic peaks are rather well-described by GEANT4. In the case of the PENELOPE simulation, when the Monte Carlo is rescaled, it is apparent that trends in both energy and angle are well represented by PENELOPE. This result is discussed quantitatively in Sec. V.

The angular dependence of the backscattered fraction can be determined by integrating the data over $q$. The result of doing so is shown in Fig. 2, and is compared with our previous results for silicon and beryllium targets.

A linear fit based on the first $20 \mathrm{keV}$ of data above the analysis cut was used to extrapolate to $50 \mathrm{eV}$ (the defined threshold for secondary electrons), so that these integrals and subsequent integrals could be compared with the current integration measurements. An additional systematic uncertainty was assigned to the extrapolation, based on differences of fit functions, and comparison to models. For $124 \mathrm{keV}$ beam energy, this extra systematic uncertainty was 5\%, resulting in a contribution due to extrapolation of typically $6 \%$ (see Table I).

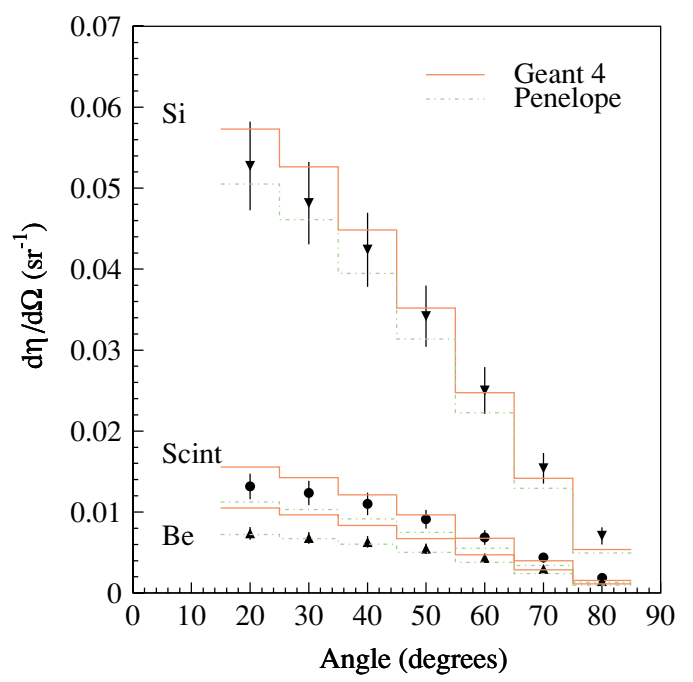

FIG. 2. (Color online) $d \eta / d \Omega$ for beryllium (triangles) and silicon (inverted triangles) targets at $E_{\text {beam }}=124 \mathrm{keV}$. Black points with error bars indicate data with total normalization systematic uncertainties shown. Red solid histogram indicates the results of the GEANT4-based Monte Carlo simulation. Green dot-dashed histogram indicates the results of the PENELOPE-based Monte Carlo simulation. No Monte Carlo scale factors are included.

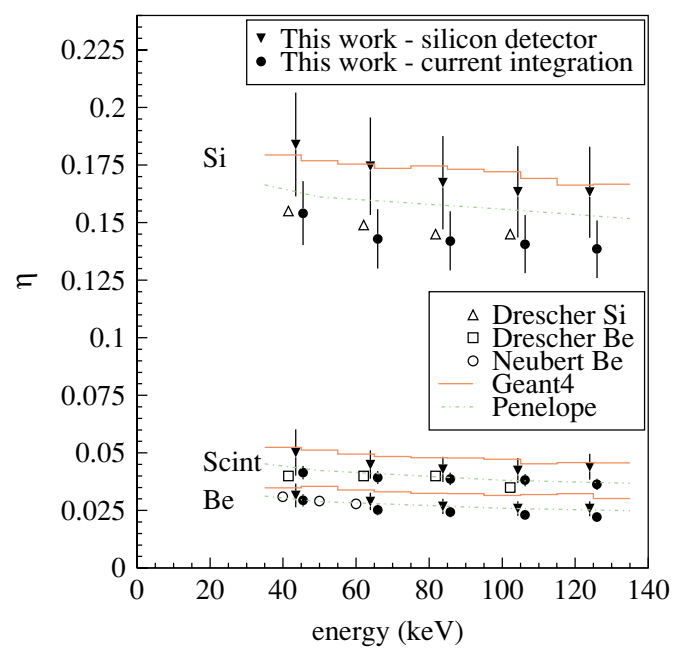

FIG. 3. (Color online) Normal incidence backscattering from $\mathrm{Be}, \mathrm{Si}$, and plastic scintillator targets. Integrated silicon detector measurements are shown by the inverted filled triangles. Current integration measurements are shown by filled circles. Total systematic uncertainties are shown and the current integration measurements are displaced by $2 \mathrm{keV}$ so that the error bars do not overlap. Previous current integration measurements $[10,11]$ are displayed. The histograms show the results of the GEANT4 (Red solid) and PENELOPE (Green dot-dashed) Monte Carlo simulations.

Figure 2 also compares the GEANT4 and PENELOPE simulations with the data. No Monte Carlo scale factors are applied for this comparison. Each Monte Carlo separately correctly predicts that the scintillator results should be larger than the beryllium results, as expected due to the larger $Z$ of the carbon nuclei in scintillator. As noted previously, the PENELOPE simulation tends to better describe trends in angle. The GEANT4 distributions are somewhat narrower compared to the data and PENELOPE. Additionally, the GEANT4 simulation gives systematically larger backscattering from each material than does the PENELOPE simulation.

The data were integrated over angle to determine the total normal-incidence backscattered fraction $\eta$. The results of this integration are shown in Fig. 3 and are compared with current integration measurements (described in Sec. IV B), with previous data, and with the models.

In Fig. 3, the total systematic uncertainty, including extrapolation to $50 \mathrm{eV}$ and extrapolation over unmeasured angles, is plotted.

Figure 3 compares the data with the GEANT4 and PENELOPE simulations. The same discrepancies in normalization are again observed. Both PENELOPE and GEANT4 adequately describe the reduction of $\eta$ as the beam energy increases.

\section{B. Current integration mode}

The results for $\eta$ based on our current integration measurements are also shown in Fig. 3.

The silicon detector measurements are found to be systematically higher than the current mode measurements; however, the two methods of are found to agree within the systematic uncertainties. In the case of the current integration method, 
this systematic uncertainty is dominated by residual correction for secondary electron collection due to the penetration through the grid of the target rod and reproducibility of the experimental results under varying conditions (see Table II). In the case of the silicon detector measurements, it is dominated by reproducibility, and by uncertainties in alignment, solid angle effects, and extrapolation to $50 \mathrm{eV}$ (see Table I).

The data are also compared with previous data on $\mathrm{Be}$ and $\mathrm{Si}$ targets due to Drescher et al. [10] and with data on Be due to Neubert et al. [11]. Both groups used current integration techniques to arrive at their results. Neubert et al. [11] in particular used a second target apparatus to study the effects of secondary electrons, as opposed to the grid used in this work. Only the subsets of their data that overlap the region 43.5 to $124 \mathrm{keV}$ are plotted. The Drescher data on Be are systematically higher than the Neubert data. As noted previously, our data tend to agree with the Neubert data on $\mathrm{Be}$, as do the data of Massoumi et al. [3,4] taken below $40 \mathrm{keV}$.

Our data on organic plastic scintillator to our knowledge are the first in this energy range. As expected, the results lie below the previous measurements on carbon (not shown), and above our data and the Neubert data on beryllium.

\section{QUANTITATIVE COMPARISON TO MODELS}

Monte Carlo to data fits including a single fit parameter, a global normalization factor, were performed for four different observables measured by our experiments (including those presented in Ref. [1]). The observables considered for fitting were $\frac{1}{N_{e}} \frac{d N}{d \Omega d q}$ at $124 \mathrm{keV}$ beam energy, $\frac{d \eta}{d \Omega}$ at $124 \mathrm{keV}$ beam energy, $\eta$ from silicon detector mode, and $\eta$ from current integration mode. The technique of $\chi^{2}$ minimization was used to constrain the fit. As mentioned in our previous work [1], point-to-point statistical and systematic uncertainties were exceedingly small relative to the overall normalization systematic. To simplify the fitting procedure, it was assumed that the point-to-point uncertainties were proportional to the global normalization uncertainty, for the purposes of evaluating $\chi^{2}$. The results of the fit are listed in Table III.

The fit results for $\frac{1}{N_{e}} \frac{d N}{d \Omega d q}(124 \mathrm{keV})$ data for scintillator are displayed in Fig. 1. Note that in our previous work [1], we did not use this fitting technique, and normalization factors were simply determined by eye. However, the normalization factors determined using the $\chi^{2}$ minimization method have a good correspondence with those numbers.

As the absolute point-to-point uncertainty was not determined precisely, the values of uncertainties on the fit parameter, and of absolute $\chi^{2}$ 's, have no meaning. Therefore only the ratio of $\chi^{2}$ s determined for the GEANT4 model, divided by that for the PENELOPE model is quoted. In other words, when taking the point-to-point uncertainty to be equivalent to the total normalization uncertainty, the value of the reduced $\chi^{2}$ was generally significantly less than unity. The exception to this was the comparison of the GEANT4 simulation to $\frac{1}{N_{e}} \frac{d N}{d \Omega d q}$ for Be targets, where an absolute reduced $\chi^{2}$ of 2.1 was seen for 188 degrees of freedom.
TABLE III. Overall scale factors and $\chi^{2}$ ratios, comparing GEANT4 to PENELOPE, under assumption of point-to-point uncertainty proportional to estimated normalization systematic uncertainty.

\begin{tabular}{lcccc}
\hline \hline Target & Observable & $\begin{array}{c}\text { GEANT4 } \\
\text { factor }\end{array}$ & $\begin{array}{c}\text { PENELOPE } \\
\text { factor }\end{array}$ & $\begin{array}{c}\chi^{2} \text { ratio } \\
(\mathrm{G} 4 / \text { Pen })\end{array}$ \\
\hline $\mathrm{Be}$ & $\frac{1}{N_{e}} \frac{d N}{d \Omega d q}(124 \mathrm{keV})$ & 0.74 & 1.10 & 3.7 \\
& $\frac{d \eta}{d \Omega}(124 \mathrm{keV})$ & 0.80 & 1.09 & 2.8 \\
& $\eta($ Si det. $)$ & 0.84 & 1.02 & 3.1 \\
& $\eta($ current int. $)$ & 0.75 & 0.91 & 2.2 \\
Si & $\frac{1}{N_{e}} \frac{d N}{d \Omega d q}(124 \mathrm{keV})$ & 1.00 & 1.08 & 1.7 \\
& $\frac{d \eta}{d \Omega}(124 \mathrm{keV})$ & 0.98 & 1.10 & 1.4 \\
& $\eta($ Si det. $)$ & 0.98 & 1.08 & 1.3 \\
& $\eta($ current int. $)$ & 0.83 & 0.91 & 1.3 \\
Scint. & $\frac{1}{N_{e}} \frac{d N}{d \Omega d q}(124 \mathrm{keV})$ & 1.07 & 1.16 & 1.8 \\
& $\frac{d \eta}{d \Omega}(124 \mathrm{keV})$ & 0.94 & 1.23 & 4.8 \\
& $\eta($ Si det. $)$ & 0.92 & 1.12 & 0.74 \\
& $\eta($ current int. $)$ & 0.80 & 0.97 & 0.38 \\
\hline \hline
\end{tabular}

Overall normalization scale factors determined for both PENELOPE and GEANT4 were generally found to agree within the normalization systematic uncertainties with unity, although deviations up to $25 \%$ are seen in certain cases. However, we note that, for observables which are not susceptible to extrapolation uncertainties, namely $\frac{1}{N_{e}} \frac{d N}{d \Omega d q}(124 \mathrm{keV})$ and $\eta$ (current int.), the PENELOPE scale factors are globally within $16 \%$ of unity.

PENELOPE generally gives a lower $\chi^{2}$ than GEANT4, indicating that the shape of the data is better described by PENELOPE. The exception is in the beam-energy dependence of $\eta$ for the scintillator data, for which GEANT4 gives a somewhat better fit. However, we believe this to be a coincidental cancellation, given that GEANT4 gives a worse description of the data for the other two observables for scintillator targets.

The reason for the superior description of the data by PENELOPE is likely due to its treatment of multiple scattering. In the case of backscattering, the multiple scattering effects are dominated by large-angle scattering. The cross section for large-angle scattering is dominated by Mott scattering. Multiple scattering algorithms, however, do not necessarily include all collisions of a given particle. Such algorithms are referred to as "condensed" algorithms, where algorithms which include all collisions are referred to as "detailed". Most particle physics simulation codes, such as GEANT4, use multiple scattering theories which are improved versions of Molière theory, and are therefore condensed algorithms requiring, e.g., step sizes to be chosen very carefully [12]. Such algorithms generally perform adequately for small angle scattering. More recently, newer multiple scattering algorithms have become available, known as "mixed" algorithms which simulate hard collisions one by one (such as large-angle Mott scattering) and use a condensed algorithm to calculate the effects of soft collisions (such as small-angle Mott scattering and electron-electron scattering). PENELOPE belongs to the mixed class of simulation codes. 
We note that aspects of PENELOPE are included in the most recent versions of GEANT4. However, the crucial aspect of PENELOPE for the correct description of backscattering, which is the mixed algorithm multiple scattering code, is to date not included in GEANT4.

\section{CONCLUSION}

Our new data on scintillator answer important questions regarding the systematic uncertainties due to backscattering for a broad range of low-energy beta spectroscopy experiments. Most importantly, the data agree well with models implemented in the GEANT4 and PENELOPE Monte Carlo codes.

Overall normalization scale factors were determined using a $\chi^{2}$ minimiziation technique. The resultant scale factors are generally found to agree within the normalization systematic uncertainties with unity. In some cases, discrepancies of up to $25 \%$ are seen. However, for observables which are not susceptible to extrapolation uncertainties, the PENELOPE scale factors are always within $16 \%$ of unity. In general, PENELOPE also gives lower $\chi^{2}$ values than GEANT4, indicating a better fit to the shape of the data in terms of energy and angle of the backscattered electrons, and in terms of beam energy. The reason for the superior description of backscattering by PENELOPE is likely due to its more accurate treatment of multiple scattering, employing a mixed algorithm treating large-angle scattering exactly, while using a condensed algorithm for small-angle scattering.

\section{ACKNOWLEDGMENTS}

We gratefully acknowledge the technical support of Robert Carr at the California Institute of Technology. This work was supported by the National Science Foundation. One of us (J. W. M) is supported by the Natural Sciences and Engineering Research Council of Canada.
[1] J. W. Martin, J. Yuan, S. A. Hoedl, B. W. Filippone, D. Fong, T. M. Ito, E. Lin, B. Tipton, and A. R. Young, Phys. Rev. C 68, 055503 (2003).

[2] P. Gérard, J. L. Balladore, J. P. Martinez, and A. Ouabbou Scanning 17, 377 (1995).

[3] G. R. Massoumi, N. Hozhabri, K. O. Jensen, W. N. Lennard, M. S. Lorenzo, P. J. Schultz, and A. B. Walker, Phys. Rev. Lett. 68, 3873 (1992).

[4] G. R. Massoumi, W. N. Lennard, P. J. Schultz, A. B. Walker, and K. O. Jensen, Phys. Rev. B 47, 11007 (1993).

[5] Eljen Technology, P.O. Box 870, Sweetwater, TX 79556. http://www.eljentechnology.com/ej-204.htm.
[6] M. J. Berger et al., the NIST ESTAR database, http://physics. nist.gov/Star (2004).

[7] S. A. Hoedl, Princeton Ph.D. thesis (unpublished) (2003).

[8] S. Agostinelli et al. (The GEANT4 Collaboration), Nucl. Instrum. Methods A 506, 250 (2003).

[9] J. Sempau et al., Nucl. Instrum. Methods B 132, 377 (1997).

[10] H. Drescher, L. Reimer, and H. Seidel, Z. Angew. Phys. 29, 331 (1970).

[11] G. Neubert and S. Rogaschewski, Phys. Stat. Sol. (a) 59, 35 (1980).

[12] This discussion is taken from the GEANT4 Physics Reference Manual July 22, 2005 version, section 7.5 Multiple Scattering, available from http://cern.ch/geant4. 